\title{
Antibiotic stewardship - is there really an app for that?
}

\author{
Authors: Sheena Bhadresha, Catherine Baldwin, Reem Santos, David Enoch and Michael E Murphy
}

\section{Aims}

Mobile apps have been developed to support antimicrobial prescribing and are likely to be familiar to the 'tech-savvy' generation of millennial-doctors. We aimed to evaluate the impact of such an app on adherence to local antimicrobial guidelines among newly qualified foundation year 1 doctors (FY1s).

\section{Methods}

In 2017, FY1s received information on antibiotics, resistance and prescribing as part of their hospital induction and were encouraged to use the newly introduced app. All antibiotics prescribed by FY1s on seven randomly selected days representative of 1 week during the first 2 months of work post-qualification in both 2016 (pre-app) and 2017 (post-app) were extracted from the electronic prescribing report including specialty, clinical indication and allergy history. Each prescription was assessed for adherence to local antimicrobial guidelines. We compared the appropriateness of antimicrobial prescribing pre- and post-app. 'Partially appropriate' was used to describe prescriptions which adhered with part of the guideline, eg co-amoxiclav monotherapy without clarithromycin for severe pneumonia. Additionally, we compared the impact on prescribing for patients with penicillin allergy for whom clear alternative guidelines exist.

\section{Results}

Fifty-one FY1s started their first post in August 2016 and 2017. All but one in the 2017 cohort reported downloading the app to their iOS or android device. The app was also available via the hospital intranet. Total app use from 127 and 84 iOS and android devices and the intranet over 2 months was 16,787 views, split 32\%, 22\% and $46 \%$ between the devices, respectively. FY1s prescribed 95 antibiotics in 76 patients in the representative 7-day sampling period in 2016 prior to introducing the app. In the same time period in 2017 after having introduced the app, there were 136 scripts in 112 patients. Allergies were present in 36 (47\%) patients, of which 10 (13\% of the total number of patients) were penicillin allergic in the pre-app data. Compared to post-app where 59 (53\%) patients were recorded to have an allergy, of these 26 (23\% of the total number of patients) were penicillin allergic. In 2016, 65 (68\%) FY1 antibiotic prescriptions adhered and 18 (19\%) partially adhered to local guidelines compared to $94(69 \%)$ and $24(18 \%)$, respectively, after the introduction of the app in 2017. In patients with documented penicillin allergy, the proportion of prescriptions which adhered to guidelines was higher after the introduction of the app, but this failed to reach statistical significance (54\% vs $72 \% ; p=0.26)$.

\section{Conclusion}

The introduction of an antimicrobial prescribing app failed to improve overall adherence to guidelines for antimicrobial prescribing among FY1 doctors in their first 2 months of starting work as qualified doctors. Adherence to guidelines improved somewhat for patients with documented penicillin allergy. Further research is required to explore why this intervention failed to positively influence behaviour change in antimicrobial prescribing, especially given the trend for investing in handheld technologies.

\section{Conflict of interest statement}

None declared. 